\section{Psychiatric morbidity in prisoners with intellectual disabilities}

Hassiotis et al ${ }^{1}$ describe an excess of probable psychosis in prisoners with intellectual disabilities $(11.3 \%$ v. $5.7 \%, P<0.01)$. We tried to replicate this finding in a large database of 21857 pre-trial reports of Dutch defendants. ${ }^{2,3}$ A diagnosis of intellectual disability $(\mathrm{IQ}<70)$ was made in 609 defendants $(2.8 \%)$. However, these individuals had fewer psychotic disorders than defendants without intellectual disability $(5.9 \%$ v. $12.7 \%, P<0.001)$. Furthermore, fewer defendants with intellectual disabilities reported misuse of hard drugs $(13.4 \%$ v. $24.6 \%, P<0.001)$ and alcohol $(16.6 \% v$. $23.1 \%, P=0.002)$ and their rate of cannabis misuse was similar to that of defendants with a normal IQ $(12.9 \%$ v. $14.2 \%$, $P=0.51)$. This again contradicts the findings of Hassiotis et al, who found more cannabis misuse and similar misuse of hard drugs and alcohol in individuals with intellectual disability.

What could explain these opposite findings? The diagnosis of probable psychosis in the Hassiotis et al study was, in $80 \%$ of the cases, based on a lay interview, and intellectual disability was defined as a low score on the Quick Test. Diagnosis in Dutch pre-trial reports is based on: (a) multiple examinations of the defendant by a psychiatrist and/or psychologist; (b) the defendant's judicial and psychiatric history, including previous examinations; (c) information from relatives; and (d) IQ tests in $88 \%$ of defendants with intellectual disabilities. As Hassiotis et al themselves suggest, their method may have led to an overestimation of the prevalence of intellectual disability (4\%). Indeed, a systematic review in 2008 showed that the prevalence of intellectual disability in prisoners ranged from 0.0 to $2.8 \%{ }^{4}$ Moreover, low scores on the Quick Test are significantly related to the prevalence of psychosis. ${ }^{5}$ Confounding of the relationship between probable psychosis and intellectual disability is therefore probable. The conclusion reached by Hassiotis et al is premature and more studies on this topic are needed.

1 Hassiotis A, Gazizova D, Akinlonu L, Bebbington P, Meltzer H, Strydom A Psychiatric morbidity in prisoners with intellectual disabilities: analysis of prison survey data for England and Wales. Br J Psychiatry 2011; 199: 156-7.

2 Vinkers $D$, Barendregt $M$, de Beurs $E$. Homicide due to mental disorder. Br J Psychiatry 2009; 194: 185

3 Vinkers DJ, de Beurs E, Barendregt M, Rinne T, Hoek HW. Pretrial psychiatric evaluations and ethnicity in the Netherlands. Int J Law Psychiatry 2010; 33: 192-6.

4 Fazel S, Xenitidis K, Powell J. The prevalence of intellectual disabilities among 12000 prisoners - a systematic review. Int J Law Psychiatry 2008; 31: 369-73.

5 Marjoram D, Gardner C, Burns J, Miller P, Lawrie SM, Johnstone EC. Symptomatology and social inference: a theory of mind study of schizophrenia and psychotic affective disorder. Cogn Neuropsychiatry 2005 10: $347-59$

David J. Vinkers, Netherlands Institute for Forensic Psychiatry and Psychology, The Netherlands, email: d.vinkers@dji.minjus.nl; Hans W. Hoek, Parnassia Bavo Psychiatric Institute, The Hague, Department of Psychiatry, University Medical Center Groningen, University of Groningen, The Netherlands, and Department of Epidemiology, Columbia University, Mailman School of Public Health, New York, USA Thomas Rinne, Netherlands Institute for Forensic Psychiatry and Psychology, The Netherlands

doi: 10.1192/bjp.199.6.514

Authors' reply: Vinkers et al have reported discrepant findings between their study and ours. First, their analysis is based on pre-trial reports, albeit detailed, whereas our study is based on a cross-sectional survey of current prisoners. Furthermore, additional variations that predetermine ascertainment and pathways through the criminal justice system must be taken into consideration in such comparisons. Our explanation of the higher rates of psychosis, one among a number of mental disorders we considered, is a combination of possible pre-existing morbidity and the impact of the environment on a vulnerable population. This relationship was mediated by current (defined as use while in prison) cannabis misuse. Second, our data on substance misuse are significant in terms of current use, as defined; lifelong use was similar between prisoners with and without intellectual disabilities. Third, the Quick Test may have led to over- or underestimation of the prevalence of intellectual disability, as we noted. There are additional arguments on this point, as the Quick Test has significant limitations: (a) we were quite conservative in the definition of intellectual disability, using not only a stringent cut-off for intellectual functioning but also poor educational attainment, and we excluded those not born in the UK, to avoid possible confounding by language-related problems; (b) according to Fazel et al, ${ }^{1}$ the pooled prevalence based on screening was $6.1 \%$ (95\% CI 5.3-7.0\%), ${ }^{2}$ therefore our calculations suggest that we have more or less identified the appropriate sample of prisoners; (c) the paper by Marjoram et $\mathrm{al}^{3}$ is, in our view, erroneously cited, as its authors discuss specifically the impact of lower IQ on participant performance in theory of mind (hinting) tasks rather than psychopathology. It should be noted that all IQ tests would be compromised if administered to acutely ill individuals. Finally, the literature suggests a common pathway between psychosis and intellectual disability, particularly in early-onset cases ${ }^{4}$ and this may be, to an extent, an underlying cause for the increased rates of psychosis. However, the cross-sectional nature of our study does not allow for further speculation on causality. In summary, prisoners with intellectual disabilities are vulnerable and may not receive adequate tailored input for their significant mental health needs. We agree that there should be further studies investigating these issues and we would like to thank Vinkers et al for their interest in pursuing this topic.

1 Fazel S, Xenitidis K, Powell J. The prevalence of intellectual disabilities among 12000 prisoners - a systematic review. Int J Law Psychiatry 2008; 31: 369-73.

2 Singleton N, Meltzer H, Gatward R. Psychiatric Morbidity among Prisoners in England and Wales. TSO (The Stationery Office), 1998.

3 Marjoram D, Gardner C, Burns J, Miller P, Lawrie SM, Johnstone EC. Symptomatology and social inference: a theory of mind study of schizophrenia and psychotic affective disorder. Cogn Neuropsychiatry 2005; 10: 347-59.

4 Hassiotis A, Strydom A, Hall I, Ali A, Lawrence-Smith G, Meltzer H, et al. Psychiatric morbidity and social functioning among adults with borderline intelligence living in private households. J Intellect Disabil Res 2008; 52: 95-106.

Angela Hassiotis, Department of Mental Health Sciences, University College London Angela London NHS Foundation Trust, The Riverside Centre, Hillingdon Hospital, Uxbridge, Middlesex; Leah Akinlonu, Hertfordshire Partnership NHS Foundation Trust, Eric Shepherd Unit, Abbots Langley; Paul Bebbington, Department of Mental Health Sciences, University College London Medical School; Howard Meltzer, Department of Health Sciences, College of Medicine, Biological Sciences and Psychology, University of Leicester; Andre Strydom, Department of Mental Health Sciences, University college London Medical School, UK

doi: 10.1192/bjp.199.6.514a

\section{Refurbishing the masked RCT design for psychological interventions}

We would like to share some important statistical pitfalls of the randomised design in masked trials of music therapy such as that conducted by Erkkilä et al. ${ }^{1}$ The randomised controlled trial (RCT) is generally considered to be the optimal design for estimating treatment efficacy in medical interventions. In a double-blind RCT, the placebo effect is equally distributed 